\title{
NUMERICAL INVESTIGATION OF PLUNGING BREAKERS OVER A POROUS ARTIFICIAL SURF REEF
}

\author{
Bjarne Jensen, DHI, bji@dhigroup.com \\ Karl-Søren Geertsen, former MSc-stud. at DTU, karlsoereng@gmail.com \\ Johan Rønby, DHI, jro@dhigroup.com \\ Simon B. Mortensen, DHI, sbm@dhigroup.com
}

\section{INTRODUCTION}

This paper presents the results of a numerical investigation of breaking waves over an artificial surf reef (ASR). In the surfing industry, it has become common to establish artificial surf reefs to enhance the surfability at popular surf locations, or to attract surfers to new locations. Besides enhancing the surfing quality, an ASR can also be seen as a submerged detached breakwater, which is a well known type of structure for coastal protection. The construction of an ASR can therefore have the additional purpose of acting as a measure for coastal protection. Hereby the ASR becomes a multi-purpose reef.

In this paper, we focus on two aspects of an ASR in relation to the effect of the porous reef: 1) a general analysis of the hydrodynamics of a plunging breaker over a reef, and 2) evaluation of specific parameters for describing the surfability and safety of the plunging breaker. The novelty of the work presented lays in the detailed inclusion of the porous reef structure in a numerical model that is applied for designing and evaluating an ASR.

\section{TEST CASE}

For this investigation of the effect of a porous reef structure we considered the ASR planned at the coastline of Palm Beach, Queensland, Australia, which was presented in Mortensen et al. (2015). The maximum width of the reef was $142 \mathrm{~m}$ and the length was $175 \mathrm{~m}$ with a mean water depth at the reef crest of $1.5 \mathrm{~m}$. The reef was proposed to be made of armour stones with a diameter of $\mathrm{d}_{50}=0.8 \mathrm{~m}$. The reef was investigated for four relevant wave conditions with $\mathrm{H}_{1 / 10}=[1.50,2.06,1.41,2.01] \mathrm{m}$ and $\mathrm{T}_{\mathrm{p}}=[9.95,9.92,9.06,13.10] \mathrm{s}$.

\section{NUMERICAL MODEL}

The numerical model was based on the Volume Averaged Reynolds Averaged Navier-Stokes equations where the resistance from the porous material was included by the extended Darcy-Forchheimer equation. The numerical method was implemented in the OpenFOAM® CFD library and validated for both two- and three-dimensional interaction with porous structures in Jensen et al. (2014).

\section{RESULTS}

The analysis of the results revolved around the effect of treating the ASR as a porous structure compared to an impermeable structure. The main outcome described the general hydrodynamics and the surfabillity. For the latter we investigated the pealing angle and speed as well as the breaker intensity.

The numerical model showed how the wave undergoes both a shoaling and a refraction process along the reef before breaking as a right-hander surf break as shown in Figure 1. The inclusion of the porous reef in the model moved the breaking point between $6 \mathrm{~m}$ and $8 \mathrm{~m}$ closer to the crest of the reef due to the energy loss associated to the flow in the porous reef. The pealing speed is important for the surfer and must be within a certain range in order for the surfer to follow the wave. The study showed that the pealing speed decreased slightly and in some cases gave a more uniform surf ride along the entire length of the ride when the porous material of the reef was taken into account.

The breaker intensity is the ratio between the length and width of the vortex tube in the breaker and it characterizes surf breaks from extreme at a low ratio and easy at a high ratio. The study showed that the breaker intensity decreased as an effect of introducing the porous material in the reef structure.

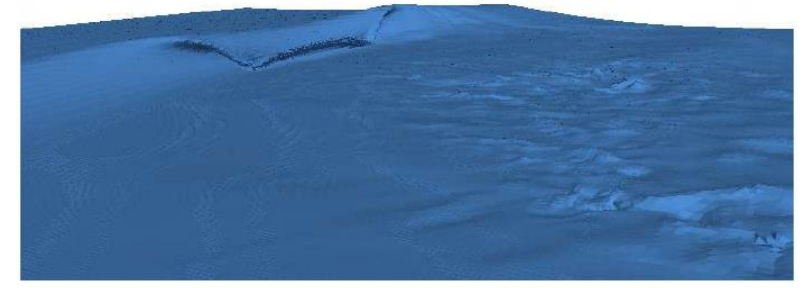

(a) Wave event 1 breaking on an impermeable reef

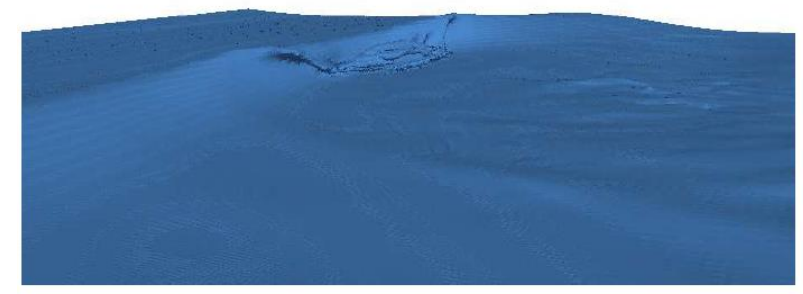

(b) Wave event 1 breaking on an porous reef

Figure 1 - Breaking wave at a) impermeable ASR and b) porous ASR.

\section{CONCLUSION}

This study showed how detailed numerical CFD modelling can be used to investigate and explain the physics of wave breaking over a porous reef in addition to aiding the design process to optimize the reef layout. The findings showed the effect of resolving the porous reef structure on the wave hydrodynamic and associated surf parameters. Hereby we extend the methods commonly applied for designing artificial surf reefs by recommending the porosity of the reef to be taken into account.

\section{REFERENCES}

Jensen B., Jacobsen, N. G., \& Christensen, E. D. (2014). Investigations on the porous media equations and resistance coefficients for coastal structures. Coastal Engineering, 84, 56-72.

Mortensen, S. B., Hibberd, W. J., Kaersgaard, K., Kristensen, S. E., Deigaard, R. \& Hunt, S. (2015), Concept Design of a Multipurpose Submerged Control Structure for Palm Beach, Gold Coast Australia. Australasian Coast and Ports Conference, Auckland. 